\title{
WISP-1 induced by mechanical stress contributes to the fibrosis and hypertrophy of ligamentum flavum via the Hedgehog-Gli1 signaling
}

\author{
Chao Sun ${ }^{1}$, Hong $\mathrm{Ma}^{1}$, Jian Yin ${ }^{1}$, Han Zhang ${ }^{1}$, and Hui Liu ${ }^{1}$ \\ ${ }^{1}$ Nanjing Medical University
}

November 9, 2020

\begin{abstract}
Background and Purpose: The ongoing chronic fibrosis and hypertrophy of ligamentum flavum (LF) is an important cause of lumbar spinal canal stenosis (LSCS). Our previous work showed that WNT1 inducible signaling pathway protein 1 (WISP-1) is a critical driver of LF fibrosis. This study was designed to explore the mechanism by which WISP-1 is activated and its underlying mechanisms in LF fibrosis. Experimental Approach: We studied the expression of Hedgehog-related proteins in human LF tissues. Cell viability, cell cycle, apoptosis rate and molecular mechanisms were evaluated in LF cells. Finally, the molecular mechanism was confirmed in vivo studies. Key Results: The results showed that Gli1 was upregulated in hypertrophic LF tissues and required for fibroblast viability and collagen expression in fibroblasts. Moreover, mechanical stretching increased WISP-1 expression in LF fibroblasts. Furthermore, WISP-1 induced fibrogenesis through Hedgehog-Gli1 pathway. This conclusion was supported by the fact that WISP-1 activated Hedgehog-Gli1 pathway in LF fibroblasts and cyclopamine attenuated the effect of WISP-1-induced fibrogenesis. WISP-1 also promoted the transition of fibroblasts into myofibroblasts via Hedgehog pathway. Importantly, hypertrophic LF rabbit model induced by mechanical stress also showed pathological change of fibrosis and more higher expression of WISP-1, Gli1 and a-SMA. Therapeutic administration of cyclopamine reduced collagen expression, fibroblast proliferation, myofibroblast differentiation and ameliorated LF fibrosis in mechanical stress-induced rabbit model.
\end{abstract}

\section{WISP-1 induced by mechanical stress contributes to the fibrosis and hypertrophy of ligamen- tum flavum via the Hedgehog-Gli1 signaling}

Chao Sun, Qinghong Ma, Jian Yin, Han Zhang, Xinhui Liu*

Department of Spine Surgery, The Affiliated Jiangning Hospital with Nanjing Medical University, Nanjing, Jiangsu 211100, China

${ }^{*}$ Corresponding author. Department of Spine Surgery, The Affiliated Jiangning Hospital with Nanjing Medical University, Nanjing, Jiangsu 211100, China.

Background and Purpose: The ongoing chronic fibrosis and hypertrophy of ligamentum flavum (LF) is an important cause of lumbar spinal canal stenosis (LSCS). Our previous work showed that WNT1 inducible signaling pathway protein 1 (WISP-1) is a critical driver of LF fibrosis. This study was designed to explore the mechanism by which WISP-1 is activated and its underlying mechanisms in LF fibrosis.

Experimental Approach: We studied the expression of Hedgehog-related proteins in human LF tissues. Cell viability, cell cycle, apoptosis rate and molecular mechanisms were evaluated in LF cells. Finally, the molecular mechanism was confirmed in vivo studies.

Key Results: The results showed that Gli1 was upregulated in hypertrophic LF tissues and required for fibroblast viability and collagen expression in fibroblasts. Moreover, mechanical stretching increased WISP-1 
expression in LF fibroblasts. Furthermore, WISP-1 induced fibrogenesis through Hedgehog-Gli1 pathway. This conclusion was supported by the fact that WISP-1 activated Hedgehog-Gli1 pathway in LF fibroblasts and cyclopamine attenuated the effect of WISP-1-induced fibrogenesis. WISP-1 also promoted the transition of fibroblasts into myofibroblasts via Hedgehog pathway. Importantly, hypertrophic LF rabbit model induced by mechanical stress also showed pathological change of fibrosis and more higher expression of WISP-1, Gli1 and a-SMA. Therapeutic administration of cyclopamine reduced collagen expression, fibroblast proliferation, myofibroblast differentiation and ameliorated LF fibrosis in mechanical stress-induced rabbit model.

Conclusion and Implications: Our study showed the mechanical stress/WISP-1/ Hedgehog signaling as a new fibrotic axis contributing to the LF hypertrophy and identified Hedgehog signaling as a therapeutic target for the prevention and treatment of LF fibrosis.

KEYWORDS : Aging; Ligamentum flavum; WISP-1; Gli1; Hypertrophy; Fibrosis

\section{Data Availability Statement}

Data available on request from the authors.

\section{1 | INTRODUCTION}

Generally, lumbar spinal canal stenosis (LSCS) mainly occurs in the aging population. Owing to the compression of the spinal cord, nerve roots, or cauda equine, it often shows symptoms of low back or/and leg pain, numbness, and intermittent claudication (Schizas et al., 2010). The development of LSCS is attributable to several pathogenic factors such as disc protrusion, facet joints degeneration, and ligamentum flavum (LF) hypertrophy. Most importantly, LF hypertrophy is considered as the important factor contributing to the development of LSCS with the age (Sakai et al., 2017).

Anatomically, the LF covers the posterior part of the dura sac, acting in limiting the range of intervertebral movement and protecting the spinal cord (Schroeder et al., 2016; Ammendolia et al., 2019). The normal LF is an elastic structure rich in elastic fibers. By contrast, the hypertrophic LF shows fibrotic changes characterized by the loss of elastic fibers and an increase of collagen fibers (Yan et al., 2018). As is known, fibrosis is the very common pathophysiological change of many chronic diseases (Rockey et al., 2015). Similarly, it is noteworthy that fibrosis is also proved to be the main pathological feature of LF hypertrophy (Yan et al., 2018; Sun et al., 2020). However, the detailed pathomechanism of LF fibrosis is not quite clear.

The CCN family is composed of six matrix proteins mainly functioning in the extracellular microenvironment between cells and plays important roles in extracellular matrix (ECM) regulation (Murphy-Ullrich \& Sage et al., 2014; Perbal et al., 2019). Among them, WISP-1, namely CCN4, has been recently studied most extensively and involved in the fibrotic process in a variety of organ systems (Yang et al., 2020; Königshoff et al., 2009). Previous work of our group showed that WISP1 was highly expressed in hypertrophied LF and closely associated with LF fibrosis (Sun et al., 2017). However, little is known about the mechanism by which WISP-1 is activated and the downstream mechanism of WISP-1 in driving activation of fibroblasts in the context of LF fibrosis has never been explored. The results presented in this study not only deepen the understanding of the role and mechanism of WISP-1 in LF fibrosis but also offer a theoretical basis for developing new drugs for LF hypertrophy.

\section{2 | EXPERIMENTAL PROCEDURES}

\subsection{Subjects}

The present study was approved by the Ethics Committee of Nanjing Medical University. From April 2018 to July 2019, 21 patients with degenerative LSCS underwent posterior decompressive laminectomy were recruited into this study. The diagnosis of LSCS was based on both clinical symptoms and radiological examinations. The inclusion criteria was as follows: age between 55 and 70 years; L4/5 level stenosis; LF hypertrophy confirmed by Magnetic Resonance Imaging (MRI). Nevertheless, patients coexisting with other diseases such as spinal tuberculosis, scoliosis, and skeletal dysplasia were excluded. The non-hypertrophied LF used in the study were obtained from age-matched patients with lumbar disc herniation (LDH) underwent 
the surgery of posterior laminectomy. The MRI was used to confirm that the LF was non-hypertrophic in this group. Informed consent was obtained from every patient prior to be enrolled in this study. The surgery was undertaken by three experienced specialists of spinal surgery in the author's hospital. The detailed characteristics of patients involved in this study were all summarized in Table 1.

\subsection{LF thickness measurement and fibrosis evaluation}

As previously stated (Sun et al., 2018), the LF thickness was assessed at the facet joint level on the T2weighted MRI from all the 42 patients. The measurement was performed on the Picture Archiving and Communication Systems (PACS) software (Nanjing Medical University, Nanjing, China). The value was measured three times for each patient by one experienced spine surgeon and the average value was considered as the LF thickness (Fig. 1A ).

All the LF samples collected for this study were only harvested from the dorsal layer of LF. LF samples were fixed in $4 \%$ paraformaldehyde phosphate buffer solution for $48 \mathrm{~h}$ and subsequently embedded in paraffin in preparation for histopathological analysis. The samples were sliced along the coronal plane for each specimen at the same level by using a paraffin microtome. After dewaxing, the sections were stained using H\&E staining, Masson's trichrome(MT) staining and elastica van Gieson (EVG) kits (JianChen, Nanjing, China). H\&E and EVG staining was used to evaluate the morphology and structure of the LF. Moreover, the degree of LF fibrosis was assessed according to the results of MT staining. The fibrotic area was quantified through Image Pro Plus 6.0 (China). As previously described (Sun et al., 2017), the grade of LF fibrosis score was classified as follows: Grade 0 indicated collagen less than $20 \%$ of the entire area; Grade 1, [?]25\% of the entire area; Grade 2, between 25\% and 50\%; Grade 3, between $50 \%$ and $75 \%$; and Grade 4, over $75 \%$ of the whole area of the sample.

\section{3 | Immunohistochemistry}

The LF specimens were fixed in $10 \%$ neutral formalin, embedded in paraffin and finally cut into sections with the thickness of $4 \mu \mathrm{m}$. After dewaxing in xylene and rehydrating in a series of alcohol solutions, the sections were firstly incubated with primary antibodies against Gli1, Shh, collagen I, collagen III and $\alpha$-SMA (Abcam) at an optimum dilution recommended by the manufacturers overnight at 4. Subsequently, these sections were incubated with the respective secondary antibody (Abcam) at room temperature. Immunohistochemical analysis was performed and visualized by confocal laser scanning microscopy (ZEISS, German).

\section{4 | Transmission electron microscopy detection}

After harvesting the LF specimens during the operation, they were immediately cut into small pieces of approximately $0.8 \mathrm{~mm}^{3}$. Subsequently, the small pieces of LF specimens were fixed in $2.5 \%$ glutaraldehyde for $3 \mathrm{~h}$. Next, the sample sections were washed 3 times for 15 minutes using phosphate-buffered saline (PBS). After dehydrating with propylene oxide 3 times for 30 minutes each time, they were embedded in Epon. Then, the specimens were cut into ultrathin sections of $70 \mathrm{~nm}$ thickness using an ultramicrotome (EM UC7, Leica, Germany). Finally, the sections were stained with $2 \%$ uranyl acetate. The morphology and structure of LF was analyzed under a transmission electron microscope (LVEM5, Delong, America).

\section{5 | LF Cell Culture}

The LF specimens obtained from patients during surgery were washed 3 times with phosphate-buffered saline (PBS, Gibco), minced into into small fragments of approximately $0.5 \mathrm{~mm}^{3}$ and digested using $0.2 \%$ type I collagenase (Gibco) at 37 for 1.5h. Subsequently, the digested pieces were washed with Dulbecco's modified Eagle's medium (DMEM, Gibco) and cultured with DMEM supplemented with $10 \%$ fetal bovine serum (Sigma), $100 \mathrm{U} / \mathrm{ml}$ penicillin and $100 \mathrm{pg} / \mathrm{ml}$ streptomycin (Sigma) in a $5 \% \mathrm{CO}_{2}$ humidified incubator at 37. The cell-culture medium was changed every two to three days. The second passage of cells was used for the subsequent experiments.

The expression levels of collagen I and III were detected by immunostaining and observed under a confocal microscope (XTL3230-DIC, Shanghai, China) to identify the cell type. The second passage of LF cells was 
cultured and stimulated with with different concentrations of recombinant human WISP-1 ranging from 0 to $150 \mathrm{ng} / \mathrm{ml}$ for $24 \mathrm{~h}$. For Hedgehog signaling inhibition experiments, $10^{6}$ cells were seeded in each well of 6 -well plates. Then, the indicated dose $(5 \mu \mathrm{M})$ of inhibitor (cyclopamine, Sigma, USA) without cytotoxicity were added in the culture medium for $24 \mathrm{~h}$. Finally, cells were collected for subsequent experiments.

\section{6 | Cell viability assays}

LF Cells were seeded in 96-well plates at $10^{5}$ cells per well and cultured at 37 under $5 \% \mathrm{CO}_{2}$ for $24 \mathrm{~h}$. Then, LF cells were incubated in $80 \mu \mathrm{l}$ of DMEM adding $20 \mu \mathrm{l} \mathrm{of} 5 \mathrm{mg} / \mathrm{ml} \mathrm{3-(4,5-dimethylthiazol-2-yl)-2,} \mathrm{5-}$ diphenyltetrazolium bromide (MTT) solution (Beyotime, Nanjing, China) for $4 \mathrm{~h}$. After the media removal, formazan crystals were dissolved in $100 \mu \mathrm{lDMSO} /$ well, and the absorbance of the solution was measured at $490 \mathrm{~nm}$. For colony-forming unit detection, the cells were pre-treated with Crystal Violet Staining Solution (Beyotime, Nanjing, China) for 10 min at 37@C. For EdU and BrDu detection, the cells were pre-treated with EdU and BrDu working solution (Beyotime, Nanjing, China) for $2 \mathrm{~h}$ at $37 @ \mathrm{C}$. Finally, they were observed under the microscope (OLYMPUS, Nanjing, China).

\section{7 | Immunofluorescence Staining}

Various treated LF cells were fixed in $4 \%$ PFA for $30 \mathrm{~min}$ at room temperature. After permeabilizing and blocking treatment, the cells were incubated with primary antibodies against a-SMA (1:500, Abcam), Gli1 (1:1000, Abcam) and WISP-1 (1:500, Abcam) overnight at 4@C. Then, cells were washed by PBS for three times and incubated with fluorescein isothiocyanate (FITC)-conjugated goat anti-rabbit antibody (1:500, Abcam) in dark conditions. Nuclei were stained with DAPI (Beyotime, Nanjing, China) for 5 min. Immunofluorescence was observed under a fluorescence microscope (OLYMPUS, Nanjing, China).

\section{8 | Transfection}

The WISP-1 and Gli1 over-expression plasmids were provided by Nanjing medical University (Nanjing, China). In brief, the human WISP-1 and Gli1 genes were then subcloned into lentiviral vector pLVXIRES-ZsGreen1 (Siri, Nanjing, China). The WISP-1 and Gli1 over-expression plasmids were transfected into human LF fibroblasts with Lipofectamine 2000 (11668019, Thermofish). After 24h transfection, cells were cultured in serum-free medium for another 24 hours. Samples were harvested for the subsequent experiment. Finally, the over-expression efficiency was tested by western blot. For gene silencing, human WISP-1- and Gli1-specific shRNAs (short hairpin RNAs; Si-WISP1: 5-CCCAAGTACTGTGGAGTTT-3 and Si-Gli1: 5-GGACAGAACTTTGATCCTT-3) were cloned into the pLL3.7 vector (Siri, Nanjing, China) as the manufacturer described. Lentiviruses containing target gene shRNA were collected and used to transfect LF fibroblasts. The blockage efficiency of shRNA was also tested by western blot.

\section{9 | Mechanical stretching stimulation of LF fibroblasts}

LF fibroblasts were seeded in a special chamber (Beyotime, Beijing, China) at $1 \times 10^{6}$ cells per chamber. The chamber had a stretching apparatus, which cloud apply a cyclic stretch (5\% and $10 \%$ elongation) for cells. In this study, $10 \%$ elongation stretching was applied for 6,12 and $24 \mathrm{~h}\left(15\right.$ cycles $\left./ \mathrm{min}, 37^{\circ} \mathrm{C}, 5 \% \mathrm{CO} 2\right)$. After stretching stimulation, total RNA and protein were isolated to measure expression of WISP-1.

\subsection{0 | RNA-seq analysis}

Total RNA was isolated from LF fibroblasts collected from LDH and LSCS patients using RNeasy Plus Micro-Kit (Takara, Dalian, China) following the manufacturer's instructions. Then, total RNA was provided to Nanjing medical university for preparing RNA-seq libraries and sequencing. The sequencing libraries were generated using the TruSeq RNA Sample Prep Kit (Illumina) as described by the manufacturer's recommendations. The libraries were sequenced on two lanes of an Illumina HiSeq X-Ten for pair-end. EdgeR was used to distinguish the differentially expressing genes by pairwise comparisons.

\section{$2.11 \mid$ RT-PCR}

According to the manufacturer's instructions, total RNA was extracted from LF tissues and cells using the 
Trizol reagent (Invitrogen) and converted to cDNA using the Reverse Transcription Synthesis Kit (Takara, Dalian, China). Then, the gene expression levels were analyzed using a Thermal Cycler Dice Real-Time system (Takara, China). After the processes of reaction, the threshold cycle $(\mathrm{Ct})$ was determined and realtive mRNA level was normalized to the GAPDH expression using the $2^{[?] \mathrm{Ct}}$ method. Designed primers and their sequences were all summarized in Table 2 and Table 3.

\subsection{2 | Western Blotting}

Proteins from LF tissues and cells were extracted by Protein Extraction Sample Kit (Sigma, USA). Subsequently, proteins were separated by SDS-PAGE, converted to a nitrocellulose membrane (Sigma, USA) by electroblotting. The membranes were blocked for $2 \mathrm{~h}$ at room temperature with $5 \%$ skim milk (Sigma) and probed with primary antibodies against WISP-1 (Abcam), Smo (Abcam), Gli1 (Abcam), Shh (Abcam), collagen I (Abcam), collagen III (Abcam), and a-SMA (Abcam,), and hybridized overnight at 4 with gentle shaking on the shaker. Finally, the membranes were incubated with 1:5000 goat anti-rabbit or anti-mouse secondary antibodies for $2 \mathrm{~h}$ at room temperature (Abcam). GAPDH (Bioworld) was used as an internal control. The intensity of each blotting band was detected using a western blotting chemiluminescence kit (Bioworld).

\subsection{3 | Rabbit LF degeneration and hypertrophy model}

All surgical and experimental procedures were approved by the ethics committee

of Nanjing Medical University. Nine New Zealand white rabbits, weighing approximately $3.0 \mathrm{~kg}$, were randomly divided into three groups in this study. The rabbits were anesthetized using $3 \%$ pentobarbital chloral hydrate $(3 \mathrm{ml} / \mathrm{kg})$ and prevented infection by injection of antibiotics $(10 \mathrm{mg} / \mathrm{kg}$ of cefazolin sodium pentahydrate; Shenzhen, China) via the ear vein. Then, the rabbits were placed into a prone position. A dorsal midline skin incision approximately $6 \mathrm{~cm}$ was performed under X-ray control. As described previously (Hayashi et al., 2017), lumbosacral fascia was cut left lateral to the mammillary process, and then discovered the gap between the multifidus and longissimus muscles. Subsequently, we detached the the multifidus from the mammillary processes to expose the lamina, the root of the transverse process and posterior edge of vertebral body. The Group A just underwent surgical exposure without fixation as a control operation $(\mathrm{n}=3)$. The Group B underwent posterolateral fusion with instrumentation (Watson locking plate; Changzhou, China) and performed additional resection of L3-4 supraspinal muscle and interspinous ligament to obtain mechanical stress concentrating on L3-4 level $(n=3)$. Briefly, 4-hole titanium locking plate was used on the left posterolateral side of L2-3 and L4-5, and a $2 \mathrm{~mm} \times 10 \mathrm{~mm}$ titanium locking screw was then inserted into each vertebra and locked to obtain mechanical stress concentrating on L3-4 level. Rabbits in Group C were treated with cyclopamine $(50 \mathrm{mg} / \mathrm{kg}$ every day, subcutaneous injection as described previously (Berman et al., 2003; Berman et al., 2002)) except for the same surgical procedure in Group B ( $\mathrm{n}=3)$. All rabbits were raised independently with free access to food and water. Three groups of rabbits were sacrificed at 12 weeks after surgery.

\subsection{4 | Statistical Analysis}

All the data were presented as mean +- standard error of the mean. Statistical analyses were done by using SPSS 17.0. Different groups were compared by using Student's t-test. Correlations among LF thickness, fibrosis score and Gli1 expression were conducted by Pearson correlation analysis. We analyzed multiple groups using one-way or two-way ANOVA. $\mathrm{P}$ value of $<0.05$ was considered to be statistically significant $\left({ }^{*} \mathrm{P}<0.05, P<0.05,{ }^{* *} P<0.01, P<0.01, P<0.01,{ }^{* * *} P<0.01\right)$.

\section{3 | RESULTS}

\section{1 | LF thickness and fibrosis score}

As is shown in Fig. 1A, the LF thickness is measured on axial T2-weighted images of MRI through the level of facet joint. In the imaging analysis, the LF has a dominating role for the load induced narrowing of LSCS. Specifically, the LF is 4 to $8 \mathrm{~mm}$-thick in patients with LSCS, contrary to the $4 \mathrm{~mm}$ or less thickness of normal 
LF (Sakai et al., 2017; Lafian \& Torralba et al., 2018). Consistent with these results, The thickness of LF was more than $4 \mathrm{~mm}$ in LSCS group. Consequently, the hypertrophic LF compressed the dural sac and nerve root and caused LSCS (Fig. 1A). In LDH group, the LF thickness was less than $4 \mathrm{~mm}$ without compression (Fig. 1A). Briefly, the mean LF thickness in the LSCS group was $4.75+-0.39 \mathrm{~mm}$, which was significantly higher than 2.57+-0.69 $\mathrm{mm}$ in the LDH group (Table 1 and Fig. 1B). Moreover, based on the Masson's trichrome (MT) staining, the fibrosis score of the dorsal side of LF in the LSCS group was 3.14+-0.85, while that of LDH group was $0.95+-0.92(\mathrm{P}<0.05)$. Furthermore, correlation analysis demonstrated that fibrosis score was positively correlated with the the LF thickness $(\mathrm{r}=0.272, \mathrm{P}<0.05)$ (Fig. 1D ), indicating that fibrosis was the main pathological feature of LF hypertrophy.

\section{2 | Structural analysis of LF tissues}

Growing evidence has demonstrated the structural disorder of LF tissues of LSCS patients (Sakai et al., 2017; Sun et al., 2020). In order to fully present the structural changes of LF tissues, we next conducted H\&E, EVG, and MT staining, and transmission electron microscope observation experiments to detect the basic structure and ultrastructure of LF. As illustrated in Figure 2A, the normal LF from LDH group were rich in elastic fibers. The fibers were arrayed in a regular order (H\&E and EVG staining, x200). Nevertheless, consistent with those previous studies (Sakai et al., 2017; Sun et al., 2020), the hypertrophic LF showed marked loss of elastic fibers in the LSCS group. The morphology of the elastic fibers was uneven, fragmented, disorganized, and partially absent (H\&E, EVG and MT staining, x200). In the MT staining, the elastic fibers were stained pink, while the collagen fibers were stained blue. In the LDH group, a large area was stained pink. But in the LSCS group, most of the area was stained blue, showing fibrotic change (MT staining, x200). As compared with LDH group, a large number of fibrosis-related proteins, including collagen I and Collagen III, were observed in LSCS group detected by immunohistochemistry.

Moreover, ultrastructural observation by the transmission electron microscopy (TEM) also showed that the LF tissues from LDH group abundantly contained elastic fibers with very few collagen fibers among them (Fig. 2B). However, there was a significant loss of elastic fibers and an increase in collagen fibers in the LSCS group. The morphology of elastic fibers on the coronal plane manily displayed long fusiform or irregular shapes in the LSCS group, while that of LDH group was round or short fusiform (Fig. 2B). The scale bar $5 \mu \mathrm{m}$ indicates $80000 \times$ magnification and the $1 \mu \mathrm{m}$ indicates $40000 \times$ magnification.

\subsection{Hedgehog-Gli1 signaling was activated in hypertrophic LF and highly associated with LF fibrosis}

To elucidate the molecular pathway involved in LF fibrosis, we focused on Hedgehog-Gli1 signaling, as this pathway was previously reported to be involved in fibrosis of various diseases (Edeling et al., 2016). As expected, gene enrichment analysis indicated that Hedgehog signaling-related proteins including Gli1 and Shh were highly elevated in fibroblasts from LSCS, suggesting the activation of Hedgehog signaling in LF (Figure 3A-B). Furthermore, PCR and western blot analyses were performed to analyze the expression of Hedgehog signaling-related proteins in LF tissues from two groups. Consistently, both the mRNA (Figure 3C) and protein (Figure 3D and 3E) expression levels of Gli1 and Shh were more higher in LF samples from LSCS group than those in the LDH group $(\mathrm{P}<0.05)$. Meanwhile, we assessed the cellular source of Gli1 and Shh on the cell by immunohistochemical analysis of LF tissues. It was found that the number of cells positive for Gli1 and Shh were significantly elevated in the LSCS group as compared to LDH group (Figure $3 \mathrm{~F}$ ). Moreover, Gli1 mRNA and protein expression was positively correlated with the LF thickness (Figure $3 \mathrm{G}, \mathrm{P}<0.05$ ) and fibrosis score, respectively (Figure 3G, $\mathrm{P}<0.05$ ). Collectively, these data suggested the activation of Sonic Hedgehog signaling in hypertrophic LF.

\section{4 | Effect of Hedgehog-Gli1 signaling on fibrogenesis in vitro}

We next elucidated the mechanism by which activated Hedgehog-Gli1 signaling promoted the fibrogenesis in vitro. It has been well demonstrated that the hedgehog pathway modulates several important aspects of function, including cell proliferation, activation and differentiation. Targeting the hedgehog pathway can be a promising direction in fibrosis treatment (Lim et al., 2018). To further confirm that the activation 
of Hedgehog-Gli1 signaling on the effect of LF cell viability, we analyzed the effect of Gli1 expression on the proliferation and apoptosis of the LF fibroblasts. As shown in the immunofluorescence staining (Fig. $4 \mathrm{~A}$ ), most of the cells were stained with collagen I or collagen III (markers of LF fibroblasts), indicating a high purity of LF fibroblasts obtained. Subsequently, we analyzed the cell viability with or without Gli1 overexpression or knockdown (Fig. 4B) using the MTS assay and flow cytometry. The data from Figures 4C showed that over-expressing Gli1 in LF fibroblasts markedly promoted cell proliferation as compared with the control group, whereas this effect was alleviated by the knockdown of Gli1. Moreover, flow cytometry suggested that LF cells over-expressing Gli1 exhibited a much lower percentage of cell apoptosis as compared to the control group (Fig. 4D). By contrast, The rate of apoptosis cell of the Gli1 knockdown LF cells was higher than that in the control group (Fig. 4D). Further study results revealed that the mechanism underlying anti-apoptosis in Gli1-overexpressing LF cells involved the inhibition of the Bax and the activation of Bcl-2 (Fig. 4E). Together, these above data suggest that activated Hedgehog-Gli1 signaling enhanced fibroblast viability by promoting cell proliferation and inhibiting cell apoptosis.

To further determine the relationship between the activation of Hedgehog-Gli1 signaling and fibrogenesis in fibroblasts, we analyzed the effect of Gli1 over-expression or knockdown on the expression of fibrosis-related proteins such as collagen I and III in LF fibroblasts. As illustrated in Figure 4E, over-expression of Gli1 significantly increased the mRNA and protein expressions of collagen I and III in LF cells (Fig. 4F). By contrast, the expression levels of collagen I and III in the Gli1 knockdown LF cells were lower than those in the control group (Fig. 4F). Furthermore, immunohistochemistry assay also demonstrated that overexpression or knockdown Gli1 increased or abrogated the expression of fibrosis-related proteins (Fig. 4G) in LF cells, suggesting that the activated Hedgehog-Gli1 signaling in LF cells promoted fibrogenesis in vitro.

\subsection{Mechanical stretching stress induces WISP-1 expression in LF cells}

Growing evidence has suggested that WISP-1 is involved in the process of fibrosis in various organs (Sharma et al., 2010; Scotton et al., 2009). In the previous study, we found that WISP-1 was markedly upregulated in hypertrophic LF tissuse and highly associated with several fibrosis-relevant factors, including collagen expression, LF thickness and fibrosis score. As is known, mechanical stress is the main cause of LF degeneration (Yoshiiwa et al., 2016; Fukui et al., 2015). Therefore, we suspected that mechanical stress may be the cause resulted in he abnormal expression of WISP-1 in hypertrophic LF tissuse and hereinafter refer to the LF fibrosis. To ascertain this, we constructed an experimental device in which repeated cyclic mechanical stretching stimulation was applied to LF fibroblasts obtained from patients with LSCS. The strength of the stretching stimulation was estimated from the ratio of stimulation-induced cellular elongation to intrinsic cellular size before stimulation. We examined whether WISP-1 mRNA levels were increased by stretching stimulation of $5 \%$ and $10 \%$ elongation for $24 \mathrm{~h}$. As expected, WISP-1 mRNA expression in LF fibroblasts was markedly elevated by stretching stimulation, and the increase in WISP-1 expression tended to depend on the strength of the stretching stimulation (Figure 5A). Furthermore, immunofluorescence staining indicated that the expression of WISP-1 increased by $6 \mathrm{~h}, 12 \mathrm{~h}$, and $24 \mathrm{~h}$ of mechnical stretching (10\% elongation, Figure 5B). Consistently, the levels of WISP-1 mRNA and protein (Figure 5C) in LF cells were elevated in a time-dependent manner under the action of mechanical stretching (10\% elongation).

\subsection{WISP-1 promotes the proliferation and inhibits apoptosis of LF fibroblasts}

Previous studies suggested that WISP-1 is highly implicated in the biological processe of affecting the proliferation and migration of fibroblasts (Sharma et al., 2010; Scotton et al., 2009; Ono et al., 2018). However, many questions remain about the precise ways in which WISP-1 regulate the LF fibroblasts and subsequently this biological process. In order to detailed mechanism of WISP-1 on fibrogenesis, we constructed LF cell lines with the over-expression or knockdown of WISP-1 (Fig. 6A). Clone formation revealed that LF cells over-expressing WISP-1 significantly increased fibroblast proliferation. By contrast, the WISP-1 knockdown inhibited the proliferation of LF fibroblasts (Fig. 6B). Furthermore, flow cytometry showed that WISP-1 promoted cell proliferation by improving the G0/G1 phase transition of LF fibroblasts. Briefly, the percentage of G0/G1 phase of LF cells with silenced WISP-1 were higher than that in the control group. Moreover, LF cells overexpressing WISP-1 exhibited a lower percentage of G0/G1 phase as compared with 
the control group (Fig. 6C). Meanwhile, flow cytometry analysis showed that WISP-1 inhibited LF cell apoptosis. Briefly, the apoptosis rate in WISP-1 over-expressing LF cells was lower than that of the control group, whereas the silencing of WISP-1 leaded to a much higher apoptosis rate as compared to the control group (Fig. 6D). Overall, these data suggested that WISP-1 enhanced LF fibroblast viability by promoting the cell proliferation and inhibiting apoptosis.

\section{7 | WISP-1 activates Hedgehog-Gli1 signaling in LF fibroblasts}

In order to elucidate the underlying mechanism by which elevated WISP-1 promoted LF fibrosis, we analyzed the effect of WISP-1 over-expression and knockdown on the expression of Hedgehog signaling-related proteins, including Gli1 and Shh, in LF cells from the LSCS patients. As a result, we found that over-expression of WISP-1 increased the levels of Gli1 and Shh in LF fibroblast, and knockdown of WISP-1 inhibited the expression of Gli1 and Shh (Fig. 7 A and B). Consistent with these results, recombinant human WISP-1 promoted Gli1 protein expression in a dose-dependent manner in WISP-1-treated LF cells (Fig. 7C).

Furthermore, we used immunofluorescence staining to investigate Gli1 expression and nucleus translocation upon the effect of WISP-1. The results revealed that Gli1 was preferentially distributed in the cytoplasm rather than in the nucleus in the control group. However, under the stimulation of WISP-1 over-expression, most Gli1 translocated to the nucleus. Moreover, Gli1 translocation was inhibited in the WISP-1 knockdown LF cells (Fig. 7D), indicating that WISP-1 could activate the Hedgehog-Gli1 pathway in LF fibroblasts.

\subsection{Cyclopamine suppresses WISP-1-induced fibrogenesis in vitro}

Subsequently, we performed an in vitro assay to confirm the role of the Hedgehog signaling in the profibrotic effect of WISP-1 on LF fibrosis. Firstly, we established the WISP-1 overexpression cell lines. As shown in Figures 8A, WISP-1 expression increased after plasmid transfection and the levels of Hedgehog signaling-related proteins such as Gli1 and Smo were significantly reduced by Hedgehog signaling inhibitor cyclopamine.

Previously, our in vitro experiment suggested that WISP-1 increased the collagen expression in human LF fibroblasts (Sun et al., 2017). Next, we sought to explore the intrinsic mechanisms by which WISP1 regulated collagen expression. Having observed that the WISP-1 activated Hedgehog-Gli1 signaling in LF cells in vitro and Gli1 over-expression abundantly enhanced the expression of collagen in the present study. We predicted that WISP-1 might increase collagen expression via the Hedgehog-Gli1 pathway. To conform this, we detected the expression of collagen with or without WISP-1 over-expression in the presence or absence of Hedgehog inhibitor (cyclopamine) in human LF cells. The protein expression detected by western blot demonstrated that Hedgehog-Gli1 signaling was a crucial adaptor for WISP-1 induced collagen expression, as the upregulation of collagen expression induced by WISP-1 was significantly attenuated by cyclopamine (Fig. 8B).

Having demonstrated that WISP-1 enhanced LF fibroblast viability, we next sought to explore the possible mechanisms. According to the results of the EdU assay, LF cell proliferation was significantly enhanced by WISP-1 over-expression. However, cyclopamine inhibited WISP-1-induced cell proliferation, suggesting that WISP-1 promoted LF cell proliferation via the Hedgehog signaling (Fig. 8C). Furthermore, flow cytometry demonstrated that the percentage of G0/G1 phase LF cells with WISP-1 over-expression was significantly lower as compared with the control. However, this response was suppressed by incubation with cyclopamine (Fig. 8D).

To confirm whether the positive effect of WISP-1 on LF cell viability is associated with apoptosis inhibition, we first examined the percentage of apoptotic cells by flow cytometry. This percentage comprised the sum of early and late apoptotic cells and was significantly reduced with WISP-1 overexpression in LF cells. However, the percentage of the apoptotic cells was significantly increased after following incubation with cyclopamine (Fig. 8E). Take together, above data suggested that WISP-1 promoted collagen expression and cell proliferation, and inhibited cell apoptosis through the Hedgehog pathway.

\section{9 | WISP-1 promotesa-SMA expression through Hedgehog signaling}


During fibrosis, the trans-differentiation of fibroblasts into myofibroblasts influences the production of ECM and secretes fibrosis related factors, which is a key cellular event that drives the fibrosis response in various tissues and organs. a-SMA is regarded as a marker of activated myofibroblasts (Mack \& Yanagita, 2015; Shu \& Lovicu, 2017; Rosenkranz, 2004). We found that the fluorescence intensity of a-SMA in LF tissues from LSCS group was significantly higher than that of LDH group (Fig. 9A), indicating that the transition of fibroblasts into myofibroblasts was also the important mechanism of LF fibrosis. It has been reported that CCN protein induces a-SMA expression in the fibrosis of many organs (Chen et al., 2019; Morales et al., 2011). Therefore, we suspected that WISP-1 might also induce a-SMA expression in LF fibroblast during fibrogenesis. In order to confirm this, we conducted a gain of function experiment in which we overexpressed WISP-1 by plasmid transfection. Similar to the WISP-1 and Gli1 expression results, we found that WISP-1 overexpression significantly increase a-SMA expression. In addition, the crucial role of WISP-1 in a-SMA expression in LF cells was further demonstrated by the loss-of-function in which we knocked down WISP-1. The results showed that WISP-1 knockdown significantly reduced a-SMA expression (Fig. 9B and 9C). Meanwhile, further evidence of the role of WISP-1 in a-SMA expression was generated from our experiments with immunofluorescence staining measuring fluorescence intensity (Fig. 9D).

As we had shown that Hedgehog-Gli1 signaling is required for WISP-1-induced fibrogenesis, we predicted that WISP-1 might also increase a-SMA expression via the Hedgehog-Gli1 pathway. To confirm this, we detected the level of a-SMA with or without WISP-1 overexpression in the presence or absence of cyclopamine in human LF fibroblasts. The protein expression of a-SMA in the LF cells detected by western blot was significantly reduced by cyclopamine (Fig. 9E). Next, the immunofluorescence staining was also perform to examine the expression level of a-SMA. As shown in Fig. 9F, the fluorescence intensity of a-SMA after WISP-1 overopression was higher in LF cells than that of control group. Meanwhile, WISP-1 knockdown in human LF cells substantially reduced the the fluorescence intensity of a-SMA. Furthermore, the increased fluorescence intensity of a-SMA induced by WISP-1 overopression was significantly suppressed by cyclopamine. These results indicated that WISP-1 induced a-SMA expression in human LF fibroblasts by targeting Hedgehog signaling.

\subsection{Cyclopamine prevents mechanical stress-induced LF fibrosis and hypertrophy in vivo}

As stated previously, we built a rabbit model in which mechanical stress was concentrated on the level of L3-4 segment with fixation of adjacent segments (L2-3 and L4-5, Fig. 10A). We found that the L3-4 level LF in group B was more thicker than that in group A (Fig. 10B). In addition, it was shown that the structure of the hypertrophic LF in rabbits was almost similar to that of the LF in humans (Fig. 10C, D) (Hayashi et al., 2017). Those results demonstrated the degeneration and hypertrophy of LF under the condition with continuously mechanical stress. Briefly, the L3-4 level LF in Group B showed a significant decrease in the density of elastic fibres, the disruption of elastic fibres and an increase in collagen fibres (Fig. 10C, D). The levels of mRNA expression in each group were evaluated by RT-PCR. The expression of fibrosis-related genes on the L3-4 level LF in Group B, including WISP-1, Gli1, Col1a2, Col2a1, and Col3a1 was significantly elevated as compared with that on the same level in group A, whereas elastin expression was significantly decreased $(\mathrm{p}<0.01)$ (Fig. $10 \mathrm{E}, \mathrm{F})$. These data indicated the activation of WISP-1/Hedgehog-Gli1 signaling in hypertrophic LF induced by mechanical stress in rabbit model, which were similar to the results observed in hypertrophic LF in patients with LSCS.

Moreover, our in vitro experiment suggested that cyclopamine suppressed WISP-1-induced fibrogenesis. Therefore, we proposed that Hedgehog signaling may serve as a therapeutic target for the prevention of LF fibrosis and hypertrophy. To confirm this hypothesis, the in vivo effect of cyclopamine was also analyzed in a rat LF hypertrophy model. Rat LF sections were evaluated using immunohistochemistry and RT-PCR. The results showed that LF thickness was significantly lower in cyclopamine-treated LF group (Group C) compared to the Group B, whereas the Group C showed approximately the same thickness as the group A (Fig. 10B). Cyclopamine treatment of LF in Group C attenuated the upregulation of fibrosis-related genes such as collagen, Gli1 and a-SMA, and the downregulation of elastin compared to the Group B (Fig. $10 \mathrm{E}, \mathrm{F})$. In addition to these changes of ECM component, we detected the cellular changes using our rabbit 
samples. The number of BrDu-positive proliferating cells was significantly higher in the Group B as compared with Group A, whereas the number of proliferating cells was reduced by cyclopamine-treated LF in Group C as compared with Group B (Fig. 10G). These data suggest that LF thickness is positively correlated with mechanical stress/WISP-1/Hedgehog-Gli1/a-SMA axis and Hedgehog-Gli1 signaling may serve as a therapeutic target for the prevention and treatment of LF fibrosis and hypertrophy.

\section{4 | DISCUSSION}

In the present study, we confirmed for the first time that mechanical stress induced WISP-1 expression in LF cells obtained from LF tissues of LSCS patients. Moreover, we demonstrated that WISP-1 induced LF hypertrophy and fibrosis by activating the Hedgehog-Gli1 pathway and aggravating ECM deposition by promoting LF cell proliferation, inhibiting cell apoptosis and inducing the transition of fibroblasts into myofibroblasts. Most importantly, inhibition of Hedgehog signaling suppressed mechanical stress-induced LF fibrosis in rabbit model, indicating that targeting Hedgehog signaling may be a novel strategy for the prevention and treatment of LF fibrosis.

Lumbar spinal canal stenosis (LSCS) is a very common disease in the elderly population often with symptoms of pain, limb numbness, or intermittent claudication, which is a serious threat to human health and life (Schizas et al., 2010; Yang et al., 2017; Yan et al., 2018). Currently, it is clear that LF hypertrophy is considered to be a major pathogenic factor for the occurrence and development of LSCS(Sakai et al., 2017). Similarly, the present study also demonstrated that the LF in LSCS group was significantly thicker as compared with LDH group. Histologically, the hypertrophic LF showed decrease of elastic fibers and collagen accumulation, suggesting fibrotic changes, which were consistent with those reported by previous studies (Yan et al., 2018; Sun et al., 2020).

Emerging evidence has suggested that WISP-1, a secreted matricellular protein belonging to the CCN family, is involved in the initiation and progression of fibrosis in various organs (Berman et al., 2003; Berman et al., 2002; Konigshoff et al., 2009). Our previous studies showed that WISP-1 expression increased in hypertrophic LF and was highly associated with LF fibrosis, suggesting that it is a critical fibrotic effector in LF fibrosis (Sun et al., 2017). However, the upstream mechanism of the abnormal expression of WISP-1 in $\mathrm{LF}$ tissue is not yet understood. Of note, it has been suggested that excessive mechanical stress commonly contributes to various pathological diseases (Wu et al., 2020; Yuan et al., 2018). As such, mechanical stress owing to segmental instability is usually occurred in patients with LSCS, and this increasing instability is considered to have a vital role in the development of LF fibrosis, finally leading to LF hypertrophy (Yoshiiwa et al., 2016; Fukui et al., 2015; Sairyo et al., 2007). Histologically, the degeneration of elastic fibers becomes accelerated and the accumulation of collagen is markedly increased because of the mechanical stress (Hur et al., 2015). Therefore, it would be of interest to examine whether mechanical stress induces WISP-1 expression during fibrogenesis. As expected, in the present study, we confirmed for the first time that mechanical stretching stress could directly induce WISP-1 expression in LF fibroblasts obtained from LSCS patient and our animal model of rabbit also demonstrated that mechanical stress induced LF fibrosis and activated WISP-1 expression in LF tissues. To explore the subsequent responses, we next focused on investigating the potential role of WISP-1 in LF fibrosis and the molecular mechanism probably included.

Previous research has clearly showed that Hedgehog signaling has been characterised as a critical mechanism underlying the persistent fibroblast activation in fibrotic diseases (Horn et al., 2012; Zerr et al., 2012; Liang et al., 2012). As is known, Gli family includes three members: namely Gli1, Gli2 and Gli3, which is a downstream transcription factor of Hedgehog pathway (Omenetti et al., 2011). It is noteworthy that Gli1 is the main transcription factor downstream of the Hedgehog pathway and can be viewed as readouts of pathway activity (Bariwal et al., 2019). In general, Gli1 is present in the cytoplasm and is in an inactive state. Once activated, Gli1 is cleaved and transported to the nucleus resulting in the transcription of Hedgehog target genes (Hegde et al., 2008). Consistently, our results also demonstrated that the Hedgehog signaling was activated during LF fibrosis. Briefly, we found that Gli1 and Shh were highly expressed in hypertrophic LF and Gli1 expression was highly related to LF fibrosis. The in vitro experiment demonstrated that activated Hedgehog-Gli1 signaling promoted LF fibroblast proliferation and collagen accumulation, and 
inhibited cell apoptosis. Furthermore, we found that WISP-1 over-expression promoted the Gli1 expression and nucleus translocation. Meanwhile, silencing WISP-1 suppressed the fibrotic effect of activated HedgehogGli1 signaling. In addition, a growing body of evidence suggests that Hedgehog inhibitor cyclopamine plays a crucial role in treating liver fibrosis by inhibiting several fibrotic genes (Kumar et al., 2016; Pratap et al., 2010). Similar to those studies, we demonstrated that cyclopamine inhibited WISP-1-induced fibrogenesis in vitro and in vivo. These results suggest that Hedgehog-Gli1 signaling was one of the targets of WISP-1 and mediated the fibrotic effect of WISP-1.

Myofibroblasts are mainly originated from the fibroblasts acting in producing a great number of ECM adhesive and structural proteins ( $\mathrm{Li} \&$ Kuemmerle, 2014). It is noteworthy that the trans-differentiation of fibroblasts into myofibroblasts is a key cellular event that drives the fibrosis response in a number of tissues and organs (Yoshiiwa et al., 2016; Fukui et al., 2015; Ono et al., 2018). The myofibroblasts are characterized by the expression of a-SMA(Zhao et al., 2018; Pinchuk et al., 2010). It has been reported that activated Hedgehog signaling promotes fibroblast-to-myofibroblast transition and fibrosis, whereas targeted inhibition of Hedgehog signalling ameliorates fibrosis in several diseases (Horn et al., 2012; Zerr et al., 2012; Liang et al., 2012). Similarly, in the present study, we demonstrated that a-SMA was upregulated in hyperthrophic LF, indicating that fibroblast-to-myofibroblast transition was also the important mechanism of LF fibrosis. Furthermore, it was found that WISP-1 promoted a-SMA expression through Hedgehog signaling as inhibition of Hedgehog signaling suppressed WISP-1-induced a-SMA expression in vitro. Consistently, in vivo inhibition of Hedgehog signaling by cyclopamine ameliorated the fibrotic effect induced by mechanical stress in rabbit. Taken together, it is concluded that mechanical stress/WISP-1/Hedgehog/a-SMA signaling is a new pro-fibrotic axis and provides a theoretical basis for the research and development of new drugs for LF fibrosis and hypertrophy.

It is generally accepted that integrins are key cell surface receptors for the CCN family (Sharma et al., 2010; Scotton et al., 2009; Ono et al., 2018). Our ongoing study is to find the corresponding receptor that are responsible for determining the selectivity of WISP-1 on Sonic Hedgehog pathway. Overall, the present data revealed the important role of the mechanical stress-WISP-1-Hedgehog axis in LF fibrosis and provided a better understanding for LF hypertrophy. The results from in vitro and in vivo experiments indicated that interfering with Hedgehog signaling may be a novel strategy for the prevention and treatment of LF fibrosis and hypertrophy.

\section{ACKNOWLEDGMENTS}

This work was supported by the National Natural Science Foundation of China (Grant No. 81802198) and Natural Science Foundation of Jiangsu Province (Grant No. SBK2019022658).

\section{CONFLICTS OF INTEREST}

The authors declare no competing interests.

\section{AUTHOR CONTRIBUTIONS}

This project was designed by C.S, and X.H.L; C.S, and H.Z. performed cell and tissue experiments; C.S., H.Z., and Q.H.M. conducted animal experiments; C.S, and X.H.L. wrote the paper. All authors have read and approved the final submitted manuscript.

\section{DECLARATION OF TRANSPARENCY AND SCIENTIFIC RIGOUR}

This Declaration acknowledges that this paper adheres to the principles for transparent reporting and scientific rigour of preclinical research as stated in the BJP guidelines for Design \& Analysis, Immunoblotting and Immunochemistry, and Animal Experimentation, and as recommended by funding agencies, publishers and other organisations engaged with supporting research.

\section{Abbreviations}

WISP-1: WNT1 inducible signaling pathway protein 1; LF: ligamentum flavum; LSCS: lumbar spinal canal 
stinosis; LDH: lumbar disc herniation; ECM: extracellular matrix; a-SMA: alpha-smooth muscle actin; MT: Masson's trichrome; EVG: Elastic van gieson.

\section{REFERENCES}

Ammendolia, C., Rampersaud, Y.R., Southerst, D., Ahmed, A., Schneider, M., Hawker, G., .. Cote, P. (2019). Effect of a prototype lumbar spinal stenosis belt versus a lumbar support on walking capacity in lumbar spinal stenosis: a randomized controlled trial. Spine J, 19, 386-394.

Bariwal, J., Kumar, V., Dong, Y., \& Mahato, R.I. (2019). Design of Hedgehog pathway inhibitors for cancer treatment. Med Res Rev, 39, 1137-1204.

Berman, D.M., Karhadkar, S.S., Hallahan, A.R., Pritchard, J.I., Eberhart, C.G., Watkins, D.N., ;;; Beachy, P.A. (2002). Medulloblastoma growth inhibition by hedgehog pathway blockade. Science, 297, 1559-1561.

Berman, D.M., Karhadkar, S.S., Maitra, A., Montes De Oca, R., Gerstenblith, M.R., Briggs, K., ... Beachy, P.A. (2003). Widespread requirement for Hedgehog ligand stimulation in growth of digestive tract tumours. Nature, 425, 846-851.

Chen, Y.Z., Sun, D.Q., Zheng, Y., Zheng, G.K., Chen, R.Q., Lin, M., .. Wu, B.Q. (2019). WISP1 silencing confers protection against epithelial-mesenchymal transition of renal tubular epithelial cells in rats via inactivation of the wnt/B-catenin signaling pathway in uremia. J Cell Physiol, 234, 9673-9686.

Edeling, M., Ragi, G., Huang, S., Pavenstädt, H., \& Susztak, K. (2016). Developmental signalling pathways in renal fibrosis: the roles of Notch, Wnt and Hedgehog. Nat Rev Nephrol, 12, 426-439.

Fukui, D., Kawakami, M., Yoshida, M., Nakao, S., Matsuoka, T., \& Yamada, H. (2015). Gait abnormality due to spinal instability after lumbar facetectomy in the rat. Eur Spine J, 24, 2085-2094.

Hayashi, K., Suzuki, A., Abdullah Ahmadi, S., Terai, H., Yamada, K., Hoshino, M., .. N Nakamura, H. (2017). Mechanical stress induces elastic fibre disruption and cartilage matrix increase in ligamentum flavum. Sci Rep, 7, 13092.

Hegde, G.V., Munger, C.M., Emanuel, K., Joshi, A.D., Greiner, T.C., Weisenburger, D.D., .. Joshi, S.S. (2008). Targeting of sonic hedgehog-GLI signaling: a potential strategy to improve therapy for mantle cell lymphoma. Mol Cancer Ther, 7, 1450-1460.

Horn, A., Palumbo, K., Cordazzo, C., Dees, C., Akhmetshina., A., Tomcik, M., .. Distler, J.H. (2012). Hedgehog signaling controls fibroblast activation and tissue fibrosis in systemic sclerosis. Arthritis Rheum, 64, 2724-2733.

Hur, J.W., Kim, B.J., Park, J.H., Kim, J.H, Park, Y.K., Kwon, T.H., \& Moon, H.J. (2015). The Mechanism of Ligamentum Flavum Hypertrophy: Introducing Angiogenesis as a Critical Link That Couples Mechanical Stress and Hypertrophy. Neurosurgery, 77, 274-281.

Konigshoff, M., Kramer, M., Balsara, N., Wilhelm, J., Amarie, O.V., Jahn, A., ... Eickelberg, O. (2009). WNT1-inducible signaling protein-1 mediates pulmonary fibrosis in mice and is upregulated in humans with idiopathic pulmonary fibrosis. J Clin Invest, 119, 772-787.

Kumar, V., Mondal, G., Dutta, R., \& Mahato, R.I. (2016). Co-delivery of small molecule hedgehog inhibitor and miRNA for treating liver fibrosis. Biomaterials, 76, 144-156.

Lafian AM, \& Torralba KD. (2018). Lumbar Spinal Stenosis in Older Adults. Rheum Dis Clin North Am, 44, 501-512.

Liang, R., Šumová, B., Cordazzo, C., Mallano, T., Zhang, Y., Wohlfahrt, T., ... Distler, J.H. (2017). The transcription factor GLI2 as a downstream mediator of transforming growth factor- $\beta$-induced fibroblast activation in SSc. Ann Rheum Dis, 76, 756-764. 
Li, C., \& Kuemmerle, J.F. (2014). Mechanisms that mediate the development of fibrosis in patients with Crohn's disease. Inflamm Bowel Dis, 20, 1250-1258.

Lim, C.H., Sun, Q., Ratti, K., Lee, S.H., Zheng, Y., Takeo, M., .. Ito, M. (2018). Hedgehog stimulates hair follicle neogenesis by creating inductive dermis during murine skin wound healing. Nat Commun, 9, 4903.

Mack, M., \& Yanagita, M. (2015). Origin of myofibroblasts and cellular events triggering fibrosis. Kidney Int, 87, 297-307.

Morales, M.G., Cabello-Verrugio, C., Santander, C., Cabrera, D., Goldschmeding, R., \& Brandan, E. (2011). CTGF/CCN-2 over-expression can directly induce features of skeletal muscle dystrophy. J Pathol, 225, 490501.

Murphy-Ullrich, J.E., \& Sage, E.H. (2014). Revisiting the matricellular concept. Matrix Biol, 37, 1-14.

Omenetti, A., Bass, L.M., Anders, R.A., Clemente, M.G., Francis, H., Guy, C.D., .. Whitington, P.F. (2011). Hedgehog activity, epithelial-mesenchymal transitions, and biliary dysmorphogenesis in biliary atresia. Hepatology, 53, 1246-1258.

Ono, M., Masaki, A., Maeda, A., Kilts, T.M., Hara, E.S., Komori, T., .. Young, M.F. (2018). CCN4/WISP1 controls cutaneous wound healing by modulating proliferation, migration and ECM expression in dermal fibroblasts via $\alpha 5 \beta 1$ and TNF $\alpha$. Matrix Biol, 68-69, 533-546.

Perbal, B. (2019). CCN proteins are part of a multilayer complex system: a working model. J Cell Commun Signal, 13, 437-439.

Pinchuk, I.V., Mifflin, R.C., Saada, J.I, \& Powell DW. (2010). Intestinal Mesenchymal Cells. Curr Gastroenterol Rep, 12, 310-318.

Pratap, A., Panakanti, R., Yang, N., Lakshmi, R., Modanlou, K.A., Eason, J.D., \& Mahato, R.I. (20110). Cyclopamine attenuates acute warm ischemia reperfusion injury in cholestatic rat liver: hope for marginal livers. Mol Pharm, 8, 958-968.

Rockey, D.C., Bell, P.D., \& Hill, J.A. (2015). Fibrosis-a common pathway to organ injury

and failure. N Engl J Med, 372, 1138-1149.

Rosenkranz, S. (2004). TGF-beta1 and angiotensin networking in cardiac remodeling. Cardiovasc Res, 63, 423-432

Sakai, Y., Ito, S., Hida, T., Ito, K., Harada, A., \& Watanabe, K. (2017). Clinical outcome of lumbar spinal stenosis based on new classification according to hypertrophied ligamentum flavum. J Orthop Sci, 22, 27-33.

Sairyo, K., Biyani, A., Goel, V.K., Leaman, D.W., Booth R, Jr., Thomas, J., ... Mohan, S.E. (2007). Lumbar ligamentum flavum hypertrophy is due to accumulation of inflammation-related scar tissue. Spine (Phila Pa 1976), 32, E340-347.

Schizas, C., Theumann, N., Burn, A., Tansey, R., Wardlaw, D., Smith, F.W., \& Kulik, G. (2010). Qualitative grading of severity of lumbar spinal stenosis based on the morphology of the dural sac on magnetic resonance images. Spine (Phila Pa 1976), 35, 1919-1924.

Schroeder, G.D., Kurd, M.F., \& Vaccaro, A.R. (2016). Lumbar Spinal Stenosis: How Is It Classified? J Am Acad Orthop Surg, 24, 843-852.

Scotton, C.J., Krupiczojc, M.A., Königshoff, M., Mercer, P.F., Lee, Y.C., Kaminski, N., .. Chambers, R.C. (2009). Increased local expression of coagulation factor $\mathrm{X}$ contributes to the fibrotic response in human and murine lung injury. J Clin Invest, 119, 2550-2563.

Sharma, S., Tantisira, K., Carey, V., Murphy, A.J., Lasky-Su, J., Celedon, J.C., .. Weiss, S.T. (2010). A role for Wnt signaling genes in the pathogenesis of impaired lung function in asthma. Am J Respir Crit 
Care Med, 181, 328-336.

Shu, D.Y., \& Lovicu, F.J. (2017). Myofibroblast transdifferentiation: The dark force in ocular wound healing and fibrosis. Prog Retin Eye Res, 60, 44-65.

Sun, C., Guan, G.P., Liu, X.F., \& Zhang, H.L. (2017). Increased expression of WISP-1 contributes to fibrosis in the hypertrophied lumber ligamentum flavum. Int J Clin Exp Pathol, 10, 1356-1363.

Sun, C., Tian, J., Liu, X., \& Guan, G.. (2017). MiR-21 promotes fibrosis and hypertrophy of ligamentum flavum in lumbar spinal canal stenosis by activating IL-6 expression. Biochem Biophys Res Commun, 490, 1106-1111.

Sun, C., Wang, Z., Tian J.W., \& Wang, Y.H. (2018). Leptin-induced inflammation by activating IL-6 expression contributes to the fibrosis and hypertrophy of ligamentum flavum in lumbar spinal canal stenosis. Biosci Rep, 38, BSR20171214.

Sun, C., Zhang, H., Wang, X., \& Liu, X. (2020). Ligamentum flavum fibrosis and hypertrophy: Molecular pathways, cellular mechanisms, and future directions. FASEB J, 2020. doi: 10.1096/fj.202000635R. Online ahead of print.

Wu, H., Yu, Y., Huang, H., Hu, Y., Fu, S., Wang, Z., .. Tang, N. (2020). Progressive Pulmonary Fibrosis Is Caused by Elevated Mechanical Tension on Alveolar Stem Cells. Cell, 180, 107-121.e17.

Yanagisawa, A., Ueda, M., Sueyoshi, T., Okada, T., Fujimoto, T., Ogi, Y., ... Mizuta, H. (2015). Amyloid deposits derived from transthyretin in the ligamentum flavum as related to lumbar spinal canal stenosis. Mod Pathol, 28, 201-207.

Yan, B., Huang, M., Zeng, C., Yao, N., Zhang, J., Yan, B., ... Wang, L. (2018). Locally Produced IGF1 Promotes Hypertrophy of the Ligamentum Flavum via the mTORC1 Signaling Pathway. Cell Physiol Biochem, 48, 293-303.

Yang, X., Wang, H., Tu, Y., Li, Y., Zou, Y., Li, G., .. Z Zhong, X. (2020). WNT1-inducible signaling protein1 mediates TGF- $\beta 1$-induced renal fibrosis in tubular epithelial cells and unilateral ureteral obstruction mouse models via autophagy. J Cell Physiol, 235, 2009-2022.

Yang, Y., Zhang, L., Dong, J., Chen, Z., Xie, P., Chen, R., .. Liu, B. (2017). Intraoperative Myelography in Transpsoas Lateral Lumbar Interbody Fusion for Degenerative Lumbar Spinal Stenosis: A Preliminary Prospective Study. Biomed Res Int, 2017, 3742182.

Yoshiiwa, T., Miyazaki, M., Kawano, M., Ikeda, S., \& Tsumura, H. (2016). Analysis of the Relationship between Hypertrophy of the Ligamentum Flavumand Lumbar Segmental Motion with Aging Process. Asian Spine J, 10, 528-535.

Yuan, J., Liu, H., Gao, W., Zhang, L., Ye, Y., Yuan, L., ... Zou, Y. (2018). MicroRNA-378 suppresses myocardial fibrosis through a paracrine mechanism at the early stage of cardiac hypertrophy following mechanical stress. Theranostics, 8, 2565-2582.

Zerr, P., Palumbo-Zerr, K., Distler, A., Tomcik, M., Vollath, S., Munoz, L.E., .. Distler, J.H. (2012). Inhibition of Hedgehog signaling for the treatment of murine sclerodermatous chronic graft-versus-host disease. Blood, 120, 2909-2917.

Zhao, X., Sun, J., Chen, Y., Su, W., Shan, H., Li, Y., .. Liang, H. (2018). lncRNA PFAR Promotes Lung Fibroblast Activation and Fibrosis by Targeting miR-138 to Regulate the YAP1-Twist Axis. Mol Ther, 26, 2206-2217.

\section{Hosted file}

Figure 1.pdf available at https://authorea.com/users/374365/articles/491894-wisp-1-inducedby-mechanical-stress-contributes-to-the-fibrosis-and-hypertrophy-of-ligamentum-flavum- 
via-the-hedgehog-gli1-signaling

\section{Hosted file}

Figure 2.pdf available at https://authorea.com/users/374365/articles/491894-wisp-1-inducedby-mechanical-stress-contributes-to-the-fibrosis-and-hypertrophy-of-ligamentum-flavumvia-the-hedgehog-gli1-signaling

\section{Hosted file}

Figure 3.pdf available at https://authorea.com/users/374365/articles/491894-wisp-1-inducedby-mechanical-stress-contributes-to-the-fibrosis-and-hypertrophy-of-ligamentum-flavumvia-the-hedgehog-gli1-signaling

\section{Hosted file}

Figure 4.pdf available at https://authorea.com/users/374365/articles/491894-wisp-1-inducedby-mechanical-stress-contributes-to-the-fibrosis-and-hypertrophy-of-ligamentum-flavumvia-the-hedgehog-gli1-signaling

\section{Hosted file}

Figure 5.pdf available at https://authorea.com/users/374365/articles/491894-wisp-1-inducedby-mechanical-stress-contributes-to-the-fibrosis-and-hypertrophy-of-ligamentum-flavumvia-the-hedgehog-gli1-signaling

\section{Hosted file}

Figure 6.pdf available at https://authorea.com/users/374365/articles/491894-wisp-1-inducedby-mechanical-stress-contributes-to-the-fibrosis-and-hypertrophy-of-ligamentum-flavumvia-the-hedgehog-gli1-signaling

\section{Hosted file}

Figure 7.pdf available at https://authorea.com/users/374365/articles/491894-wisp-1-inducedby-mechanical-stress-contributes-to-the-fibrosis-and-hypertrophy-of-ligamentum-flavumvia-the-hedgehog-gli1-signaling

\section{Hosted file}

Figure 8.pdf available at https://authorea.com/users/374365/articles/491894-wisp-1-inducedby-mechanical-stress-contributes-to-the-fibrosis-and-hypertrophy-of-ligamentum-flavumvia-the-hedgehog-gli1-signaling

\section{Hosted file}

Figure 9.pdf available at https://authorea.com/users/374365/articles/491894-wisp-1-inducedby-mechanical-stress-contributes-to-the-fibrosis-and-hypertrophy-of-ligamentum-flavumvia-the-hedgehog-gli1-signaling

\section{Hosted file}

Figure 10.pdf available at https://authorea.com/users/374365/articles/491894-wisp-1-inducedby-mechanical-stress-contributes-to-the-fibrosis-and-hypertrophy-of-ligamentum-flavumvia-the-hedgehog-gli1-signaling

\section{Hosted file}

Table 1.pdf available at https://authorea.com/users/374365/articles/491894-wisp-1-inducedby-mechanical-stress-contributes-to-the-fibrosis-and-hypertrophy-of-ligamentum-flavumvia-the-hedgehog-gli1-signaling 


\section{Hosted file}

Table 2.pdf available at https://authorea.com/users/374365/articles/491894-wisp-1-inducedby-mechanical-stress-contributes-to-the-fibrosis-and-hypertrophy-of-ligamentum-flavumvia-the-hedgehog-gli1-signaling

\section{Hosted file}

Table 3.pdf available at https://authorea.com/users/374365/articles/491894-wisp-1-inducedby-mechanical-stress-contributes-to-the-fibrosis-and-hypertrophy-of-ligamentum-flavumvia-the-hedgehog-gli1-signaling 